Article

\title{
An Improved Adaptive-Torque-Gain MPPT Control for Direct-Driven PMSG Wind Turbines Considering Wind Farm Turbulences
}

\author{
Xiaolian Zhang ${ }^{1, *}$, Can Huang ${ }^{2}$, Sipeng Hao ${ }^{1}$, Fan Chen ${ }^{1}$ and Jingjing Zhai ${ }^{1}$ \\ 1 School of Electric Power Engineering, Nanjing Institute of Technology, Nanjing 211167, China; \\ hspnj@sohu.com (S.H.); fanchen_nj@163.com (F.C.); zhaijj@njit.edu.cn (J.Z.) \\ 2 Department of Electrical Engineering and Computer Science, The University of Tennessee, Knoxville, \\ TN 37996, USA; chuang16@utk.edu \\ * Correspondence: zhangx1530@163.com; Tel.: +86-139-5177-1281
}

Academic Editor: Frede Blaabjerg

Received: 9 October 2016; Accepted: 15 November 2016; Published: 22 November 2016

\begin{abstract}
Maximum power point tracking (MPPT) plays an important role in increasing the efficiency of a wind energy conversion system (WECS). In this paper, three conventional MPPT methods are reviewed: power signal feedback (PSF) control, decreased torque gain (DTG) control, and adaptive torque gain (ATG) control, and their potential challenges are investigated. It is found out that the conventional MPPT method ignores the effect of wind turbine inertia and wind speed fluctuations, which lowers WECS efficiency. Accordingly, an improved adaptive torque gain (IATG) method is proposed, which customizes adaptive torque gains and enhances MPPT performances. Specifically, the IATG control considers wind farm turbulences and works out the relationship between the optimal torque gains and the wind speed characteristics, which has not been reported in the literature. The IATG control is promising, especially under the ongoing trend of building wind farms with large-scale wind turbines and at low and medium wind speed sites.
\end{abstract}

Keywords: wind energy conversion system (WECS); wind turbine; maximum power point tracking (MPPT); adaptive torque gain control; turbulence intensity

\section{Introduction}

Wind power generation, as an important clear energy resource of modern power grids, has been developing rapidly over the past several years [1-14]. It is reported that the global wind power capacity increased from $370 \mathrm{GW}$ in 2014 to $432 \mathrm{GW}$ in 2015, representing a 17\% annual growth rate [15]. It is also anticipated that the global wind power market will continue to grow steadily in the coming years $[16,17]$. In practice, wind energy conversion systems (WECSs) are commonly used to convert wind power to electric power and further deliver the electricity to power grids. It is crucial to increase WECS efficiency to make wind power generation reliable and profitable.

Maximum power point tracking (MPPT) plays a decisive role in WECS efficiency. It was reported in 2004 that more than $50 \%$ of annual power capacity of a typical wind turbine came from MPPT [18,19]. Now, the percentage should grow, since an increasing number of wind turbines are being installed at low and medium wind speed sites [20]. Conventional MPPT control methods can be roughly divided into three categories: tip-speed ratio (TSR) control, hill-climb searching (HCS) control, and power signal feedback (PSF) control. Those control methods are well reviewed in [1,3,21], and the PSF control is viewed as the most favorable one for large-scale WECSs (e.g., multi-MW wind turbines). The PSF control typically tracks the maximum power points via a turbine characteristic curve or a lookup table, and captures the maximum wind power through adjusting electrical power or torque. 
However, the actual maximum power points are hard to achieve. A study of National Renewable Energy Laboratory (NREL) shows that a 5\% error between real wind power coefficient curves and ideal ones commonly exists in the PSF control. This error lowers MPPT performances and leads to $1 \%-3 \%$ energy loss, which is considered significant in wind energy industries $[18,19]$. Some studies further point out that the error derives from the effect of wind turbine inertia and wind speed fluctuations [22-25]. To be specific, the PSF control views MPPT as a static process and assumes that a series of maximum power points can be tracked quickly. However, the real maximum power points are difficult to capture, especially for multi-MW wind turbines and/or under turbulences (e.g., the wind speed has low mean and high turbulent values). Hence, the effect of wind turbine inertia and wind farm turbulences should be considered in MPPT design. This becomes increasingly important, especially under the ongoing trend of building wind farms with large-scale wind turbines and at low and medium wind speed sites [20-25].

Furthermore, MPPT in essence is a type of tracking problem, i.e., $V_{r e f}=V_{0}+a t$, and MPPT performances are related to three factors: the reference speed $V_{\text {ref, }}$, the initial speed $V_{0}$, and the acceleration or deceleration $a$. The majority of previous studies improve MPPT performances through tuning the acceleration or deceleration. For instance, a decreased torque gain (DTG) control to tune electrical torque gains is proposed in [26] and an adaptive torque gain (ATG) control to search the optimal gain is presented in $[18,19]$. These two methods are both based on PSF methods and further modified in $[23,27,28]$, which adaptively adjust electrical torques to improve WECS efficiency. In addition, a few recent studies have enhanced MPPT performances through regulating the initial speed, and they make use of historical wind speed data and implement the intelligent initial speed with encouraging results $[22,29,30]$. Nevertheless, the previous control methods have limitations. They ignore the effect of wind turbine inertia and/or wind speed fluctuations, thus lowering MPPT performances [31,32].

In this paper, the potential issues of PSF control, DTG control, and ATG control are investigated, and an improved ATG (IATG) control is proposed. The IATG control especially considers the effect of wind farm turbulences, which is likely more realistic for practicing engineers.

The rest of this paper is organized as follows: Section 2 introduces classic WECS models and conventional MPPT methods, including the PSF control, DTG control, and ATG control; and Section 3 investigates the potential challenges of the conventional MPPT methods and proposes an improved MPPT method. Furthermore, the simulation results and comparative analysis are presented in Section 4, and conclusions are drawn in Section 5.

\section{Conventional MPPT (Maximum Power Point Tracking) Methods}

In this section, some background information is introduced, including classic WECS models and conventional MPPT methods. A variety of WECS configurations can be broadly classified into four types: Type 1 (fixed-speed WECS), Type 2 (limited-variable-speed WECS), Type 3 (variable-speed with partial power electronic conversion WECS), and Type 4 (variable-speed with full power electronic conversion WECS) [33-37]. In recent years, an increasing number of wind farms are using the Type 4 WECS, specifically the permanent magnetic synchronous generator (PMSG) based WECS, due to its high efficiency, gearless construction, light weight quality, and self-excitation features [21,34-36]. Thus, the PMSG-based WECS is studied here.

\subsection{WECS Models}

A typical PMSG-based WECS is shown in Figure 1, in which wind power is transformed to mechanical power via a wind turbine, and further converted to electric power through a generator [37-40]. The wind power $P_{\text {wind }}$ and the mechanical power $P_{m}$ can be expressed as

$$
P_{\text {wind }}=\frac{\rho \pi R^{2} v^{3}}{2}
$$




$$
\begin{gathered}
P_{m}=\frac{\rho \pi R^{2} v^{3}}{2} \cdot C_{p}(\lambda, \beta) \\
C_{p}(\lambda, \beta)=0.5176\left(116 / \lambda_{i}-0.4 \beta-5\right) e^{-21 / \lambda_{i}}+0.0068 \lambda \\
\frac{1}{\lambda_{i}}=\frac{1}{\lambda+0.08 \beta}-\frac{0.035}{\beta^{3}+1}
\end{gathered}
$$

where $\rho$ is the air density, $R$ is the turbine radius, $v$ is the wind speed, $C_{p}$ is the power coefficient, $\lambda=\omega R / v$ is the TSR, $\omega$ is the turbine angular speed, and $\beta$ is the pitch angle.

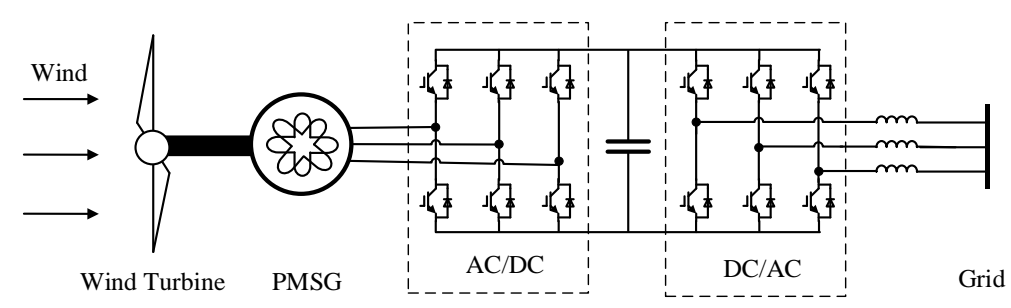

Figure 1. A typical wind energy conversion system.

In addition, the turbine and generator are directly connected by a shaft, and they have the following relationship $[22,29,30]$ :

$$
\begin{gathered}
\dot{\omega}=\left(T_{m}-T_{e}\right) / J \\
T_{m}=P_{m} / \omega=\frac{\rho \pi R^{5} \omega^{2}}{2 \lambda^{3}} C_{p}(\lambda, \beta) \\
T_{e}= \begin{cases}T_{\max }=P_{\max } / \omega & v_{c i} \leq v<v_{\text {rat }} \\
T_{\text {rat }}=P_{\text {rat }} / \omega & v_{\text {rat }} \leq v<v_{c o} \\
0 & \text { otherwise }\end{cases}
\end{gathered}
$$

where $T_{m}$ is the mechanical torque (i.e., turbine torque), $T_{e}$ is the electrical torque (i.e., generator torque), and $J$ is the turbine's moment of inertia. Specifically, $v_{c i}, v_{r a t}$, and $v_{c o}$ are the cut-in wind speed, rated wind speed, and cut-out wind speed, which divide the WECS into two operating regions as shown in Figure 2. In region I, the WECS should capture the maximum power $P_{\max }$, and in region II, the WECS should extract the rated power $P_{\text {rat }}$.

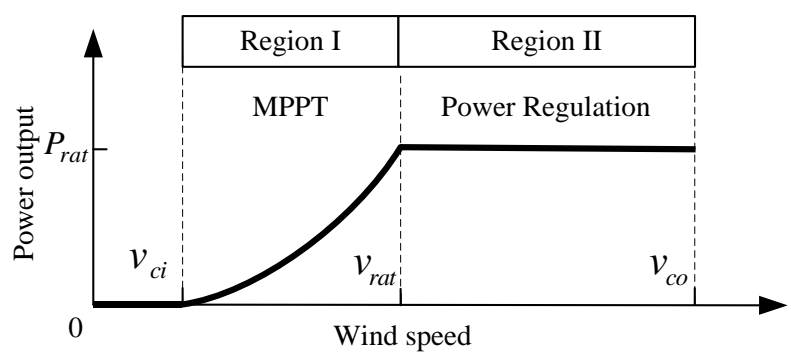

Figure 2. An ideal power curve for a variable-speed variable-pitch wind turbine.

\subsection{MPPT Methods}

The conventional MPPT methods can be classified into three categories: TSR control, HCS control, and PSF control $[1,3,21]$. In general, the TSR control regulates the generator rotor speed $\omega$ to maintain the optimal TSR and extract the maximum power. The HCS control continuously searches for the maximum power points of a wind turbine through an increase or a decrease of $\omega$ in each step. In the PSF control or its variation named optimal torque (OT) control, as shown in Figure 3, a wind turbine generates the maximum power $P_{\max }$ when it operates at the maximum power coefficient point $C_{\text {pmax }}=\left(\lambda_{\text {opt }}, \beta_{\text {opt }}\right)$, where $\lambda_{\text {opt }}=\omega_{\text {opt }} R / v$ and $\beta_{\text {opt }}=0$. 


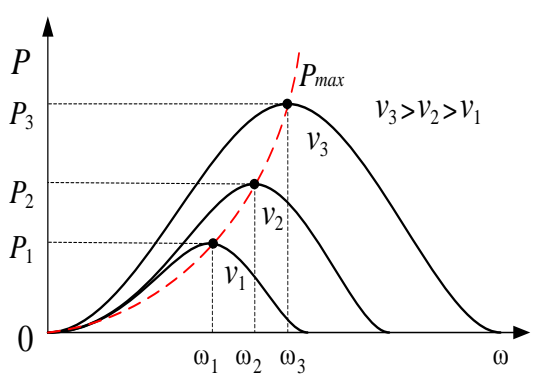

(a)

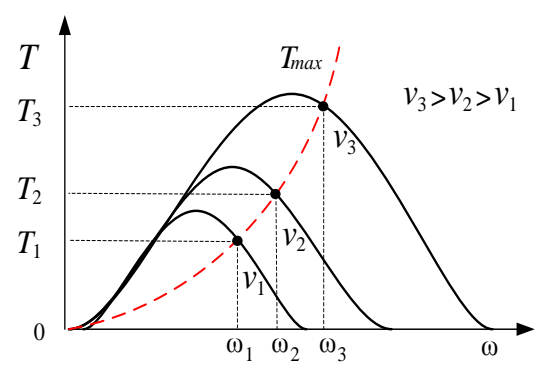

(b)

Figure 3. Wind turbine characteristic curves: (a) typical wind turbine power-speed characteristic curves for different wind speeds and the optimal power curve; and (b) typical wind turbine torque-speed characteristic curves for different wind speeds and the optimal torque curve.

The PSF control and OT control are the most commonly used MPPT control methods in practice, especially the ones with multi-MW wind turbines [2,21]. The maximum power output in the PSF control can be written as

$$
P_{\max }=\frac{\rho \pi R^{5} C_{p \max }}{2 \lambda_{o p t}^{3}} \omega^{3}=k_{o p t} \omega^{3}
$$

Note that the optimal coefficient $k_{\text {opt }}$ in Equation (8) and the wind turbine characteristic curve can be obtained through simulations and field tests [27]. The knowledge of $k_{\text {opt }}$ in the PSF/OT control is normally assumed pre-known and stored in a look-up table, and the MPPT can be performed simply and quickly [21]. In addition, the characteristic of fractional average power $\eta_{\text {favg }}$ is often used to evaluate MPPT efficiency, which is defined as the ratio of the mean captured power to the mean wind power as follows [22]:

$$
\eta_{\text {favg }}=\frac{1}{n} \sum_{i=1}^{n} P_{m}(i) / \frac{1}{n} \sum_{i=1}^{n} P_{\text {wind }}(i)=\sum_{i=1}^{n} C_{p}(i)
$$

where $n$ is the sampling times in one sampling period.

$$
T_{e}=K_{d} \cdot k_{o p t} \omega^{2}=K_{d} T_{\max }
$$

The DTG control and ATG control improve the PSF control by multiplying a gain coefficient $K_{d}\left(K_{d}<1\right)$ on the electrical torque in Equation (10). In this way, the generator can obtain a lower deceleration but a higher acceleration and can track the maximum power points more efficiently. This is because the wind power generation is cubic to the wind speed, and the wind power harvest during deceleration (i.e., under low wind speeds) is generally smaller than the one during acceleration (i.e., under high wind speeds) $[18,19,22]$. In addition, there exists an optimal gain $K_{\text {dopt }}$, which contributes to the maximum fractional average power $\eta_{\text {favg }}^{\max }$ as shown in Figure 4 .

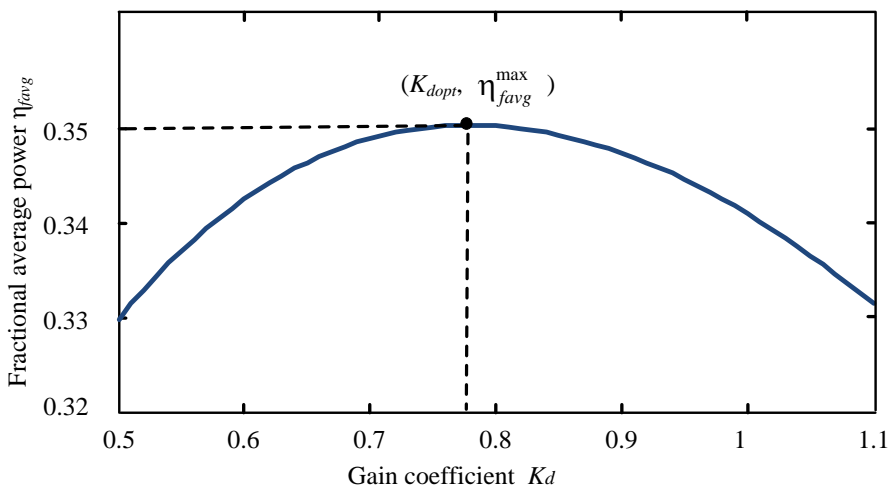

Figure 4. Optimal torque gain curve. 
Specifically, the DTG control acquires the optimal gain $K_{\text {dopt }}$ from empirical studies and assumes it as constant, and the ATG control obtains $K_{\text {dopt }}$ through perturbation and observation ( $P$ \& $\mathrm{O}$ ) schemes. The ATG control performs an iterative search of $K_{\text {dopt }}$, in which the electrical torque is adjusted as Equation (11) and $K_{d}$ is updated through iterations as Equation (12) [18,19]. Consequently, a brief review of the five MPPT methods is presented in Table 1 [1,3,18-22]:

$$
\begin{gathered}
T_{e}(\omega)= \begin{cases}0 & \omega \leq \omega_{\text {bgn }} \\
K_{d}(k+1) T_{\max } & \omega>\omega_{\text {bgn }}\end{cases} \\
\begin{cases}K_{d}(k+1)= & K_{d}(k)+\Delta K_{d}(k+1) \\
\Delta K_{d}(k+1)= & \gamma_{k d} \operatorname{sign}\left[\Delta K_{d}(k)\right] \\
& \times \operatorname{sign}\left[\Delta \eta_{\text {favg }}(k)\right] \sqrt{\left|\Delta \eta_{\text {favg }}(k)\right|} \\
\Delta K_{d}(k)= & K_{d}(k)-K_{d}(k-1) \\
\Delta \eta_{\text {favg }}(k)= & \eta_{\text {favg }}(k)-\eta_{\text {favg }}(k-1)\end{cases}
\end{gathered}
$$

where $k$ is the iteration index, $\gamma_{k d}$ is the coefficient of step adjustment, and $\omega_{\text {bgn }}$ is the starting rotor speed.

Table 1. A brief review of maximum power point tracking (MPPT) methods.

\begin{tabular}{cccc}
\hline MPPT Methods & Tracking Speed & Tracking Reference & Tracking Accuracy \\
\hline TSR Control & High & $\begin{array}{c}\omega_{\text {ref }}=\lambda_{\text {opt }} v / R \text { and } \lambda_{\text {opt }} \text { is } \\
\text { assumed to be pre-known }\end{array}$ & Low \\
\hline HCS Control & Low & $\begin{array}{c}\text { Updated on-line and with no } \\
\text { prior-knowledge }\end{array}$ & High \\
\hline PSF Control & High & $\begin{array}{c}P_{\text {ref }}=k_{\text {opt }} \omega^{3} \text { and } k_{\text {opt }} \text { is } \\
\text { assumed to be pre-known }\end{array}$ & Medium \\
\hline DTG Control & High & $\begin{array}{c}P_{r e f}=K_{d} k_{o p t} \omega^{3} \text { and } K_{d} \text { is } \\
\text { assumed to around } 0.8-0.9\end{array}$ & Medium \\
\hline ATG Control & Medium & $\begin{array}{c}P_{r e f}=K_{d} k_{o p t} \omega^{3} \text { and } K_{d} \text { is } \\
\text { updated on-line }\end{array}$ & High \\
\hline
\end{tabular}

Note: TSR: tip-speed ratio; HCS: hill-climb searching; PSF: power signal feedback; DTG: decreased torque gain; ATG: adaptive torque gain.

\section{Improved MPPT Method}

\subsection{Challenges of DTG Control and ATG Control}

As aforementioned, the DTG control views the optimal gain $K_{\text {dopt }}$ as pre-known and uses the fixed $K_{\text {dopt }}$ based on empirical studies. However, the optimal gain is hard to obtain in reality, and the constant value will become invalid with aging.

To address this issue, the ATG control customizes the $\mathrm{P} \& \mathrm{O}$ scheme (perturbation as $\Delta K_{d}$ and observation as $\Delta \eta_{\text {favg }}$ ) to adaptively update the optimal gain. Theoretically, the searching direction is determined by the variation of fractional average power $\Delta \eta_{\text {favg }}$, and the observation $\Delta \mathfrak{\eta}_{\text {favg }}$ is decided by the perturbation $\Delta K_{d}$. In reality, since the real-time wind speed is the input of the whole system, the observation $\Delta \eta_{\text {favg }}$ is impacted not only by the perturbation $\Delta K_{d}$, but also by the variable wind speed.

Hence, the ATG control ignores the effect of wind speed fluctuations, meaning that it may go to search in the wrong direction and fail to find out the exact optimal gain, especially under rapidly-changing turbulences. For example, as shown in Figure 5, in the normal ATG control, the WECS is expected to climb uphill and arrive at the $K_{\text {dopt }}$ point (e.g., the expected $\Delta \eta_{\text {favg }}(k)$ is positive, the WECS climbs uphill, and the searching process is A-B-C); in reality, the WECS may experience a wind speed change and mistakenly travel downhill (e.g., the real $\Delta \eta_{\text {favg }}(k)$ is negative, the WECS travels downhill, 
and the searching process is $\left.A-B^{\prime}-C^{\prime}\right)$. The ATG control is blind to atmospheric changes, and its $\mathrm{P} \& \mathrm{O}$ rules may lead to MPPT failures.

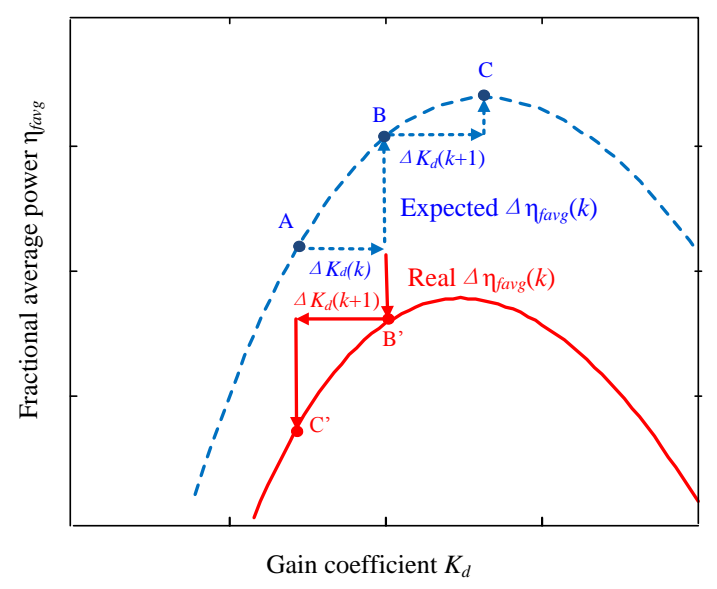

Figure 5. ATG control losing its tractability under changing wind speed and traveling downhill instead of climbing uphill, where the solid and dashed lines are the optimal torque curves under different wind speeds.

To further investigate the above issue, the ATG control under two different wind speed profiles is tested. Here, the wind speed is featured with two components, including the mean wind speed $v_{m}$ and the turbulence intensity $T_{i}$. The two wind speed profiles as shown in Figure 6 are with the same mean wind speed but different turbulences (i.e., Class-A and Class- $C$ turbulences, which represent higher and lower turbulence intensities defined by IEC-614000-1, respectively). Additionally, wind speeds I and II both last $600 \mathrm{~min}$, in which the ATG control runs 20 min per iteration and totally performs over 30 times. In this case, the tractability of the ATG control is tested.
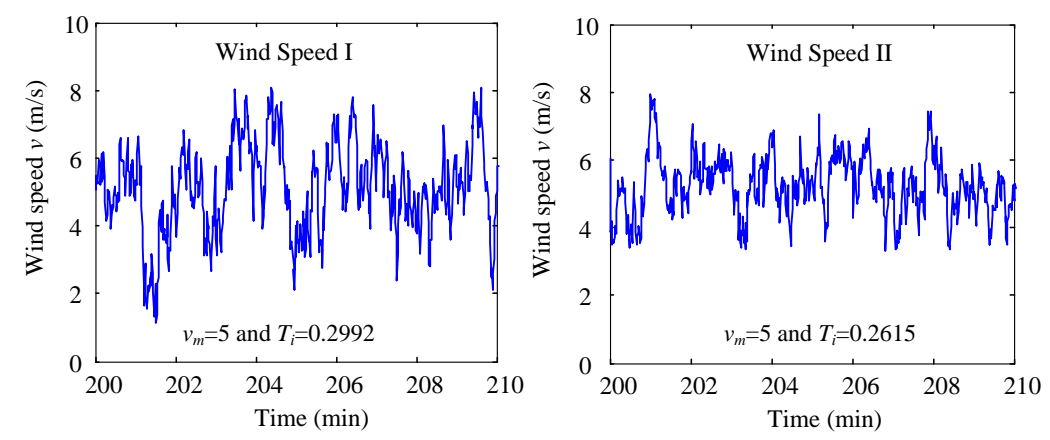

Figure 6. Wind speed profiles including wind speed I and wind speed II.

TEST: The gain coefficients obtained by the $k$ th and $(k+1)$ th iterations are denoted as $K_{d}(k)$ and $K_{d}(k+1)$, respectively, while the real optimal gain coefficients under the $k$ th and $(k+1)$ th wind speeds are denoted as $K_{d_{-} \text {real }}(k)$ and $K_{d_{-} \text {real }}(k+1)$, respectively. $K_{d_{-} \text {real }}(k)$ and $K_{d_{-} \text {real }}(k+1)$ are calculated by traversal. In addition, the state "flag" below is used to suggest the correctness of search direction.

$$
\begin{array}{ll}
\text { If } \quad & \operatorname{sign}\left(K_{d}(k+1)-K_{d}(k)\right)=\operatorname{sign}\left(K_{d \_ \text {real }}(k+1)-K_{d \_ \text {real }}(k)\right) \\
& \text { flag }=1 ; \\
\text { else } & \text { flag }=0
\end{array}
$$

As a result, the probability of an incorrect search of the ATG control $P_{\text {wrong }}$ is counted, and the values of fractional average power under wind speeds I and II are calculated. The corresponding results are presented in Table 2. 
Table 2. ATG control performances under wind speeds I and II.

\begin{tabular}{ccc}
\hline Wind Speed Profile & $\boldsymbol{P}_{\text {wrong }}$ & $\boldsymbol{\eta}_{\text {favg }}$ \\
\hline Wind Speed I & $48.28 \%$ & 0.3732 \\
Wind Speed II & $41.38 \%$ & 0.3860 \\
\hline
\end{tabular}

It is found that the ATG control often goes searching in the wrong direction, which lowers MPPT efficiency; this issue gets worse with increasing turbulence intensity.

\subsection{Improved ATG control}

In this paper, an IATG control is proposed, with the consideration of wind speed fluctuations. It primarily consists of two parts as shown in Figure 7.

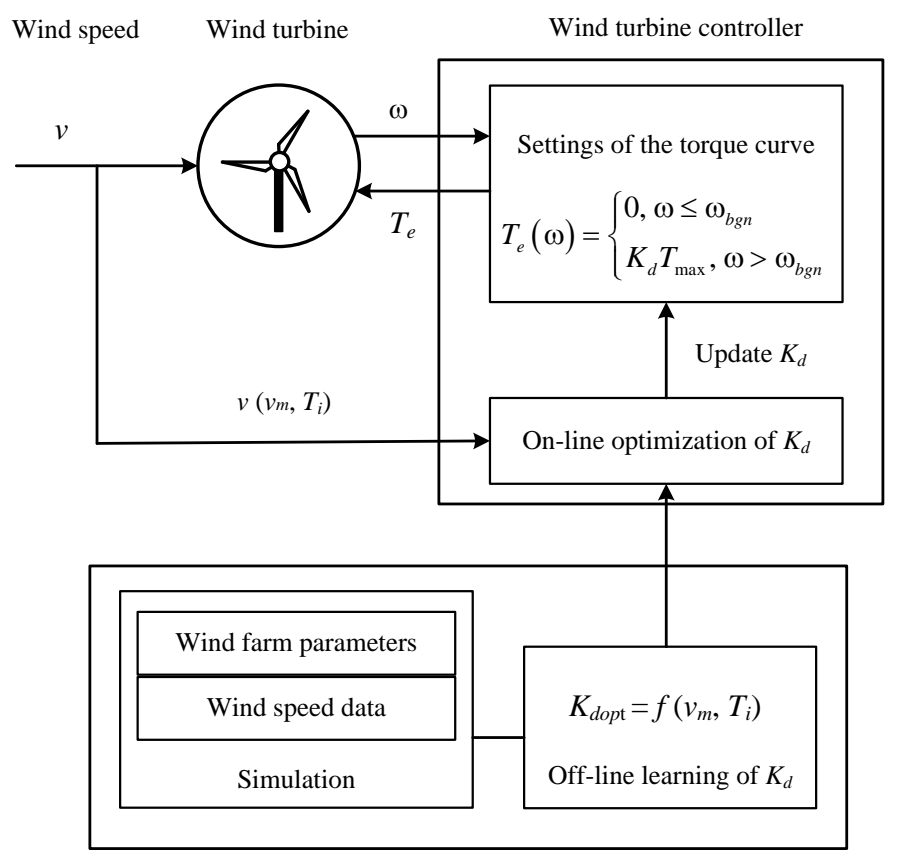

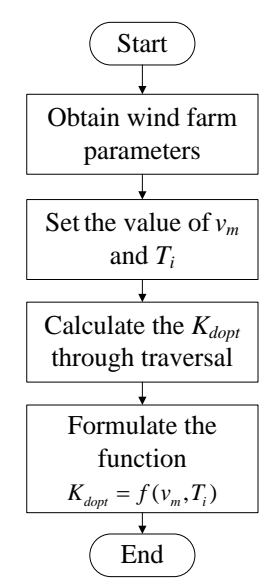

(b) (a)

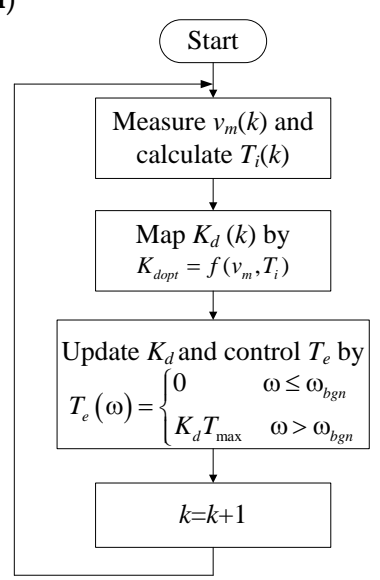

(c)

Figure 7. IATG (improved adaptive torque gain) control: (a) schematic diagram; (b) flow diagram of off-line learning; and (c) flow diagram of on-line optimization.

The first part works on the off-line learning of the relationship between optimal gain coefficients and wind speed characteristics (i.e., mean wind speed and turbulence intensity). For a given wind farm, 
we utilize a great amount of historical wind speed data and the corresponding optimal gain coefficients, and obtain the relationship between optimal gain coefficients and wind speed characteristics via advanced statistical and fitting techniques. Details is given as follows:

Step 1.1 Data Profiles: Collect wind farm parameters and create a complete set of wind speed data with wind speed emulation techniques [41]. Here, the low and medium mean wind speeds $v_{m}$ range from $4 \mathrm{~m} / \mathrm{s}$ to $7 \mathrm{~m} / \mathrm{s}$ with the sampling step of $0.2 \mathrm{~m} / \mathrm{s}$, the sampling period of $20 \mathrm{~min}$, and the integral length scale as 100. According to IEC-61400-1 standard, the turbulence intensities $T_{i}$ of three categories A, B, and C, range from 0.18 to 0.35 with the changing step of 0.01 . As a result, there are 288 wind speed profiles in total.

Step 1.2 Simulation: Perform the WECS simulation under different wind speed profiles and obtain the corresponding optimal gain coefficient $K_{\text {dopt }}$ through traversal.

Step 1.3 Formulation: Perform the statistics of the average wind speed $v_{m}$, the turbulence intensity $T_{i}$, and the corresponding optimal gain $K_{\text {dopt }}$, and formulate the function $K_{\text {dopt }}=f\left(v_{m}, T_{i}\right)$ using least square algorithms.

The second part deals with the on-line optimization of the optimal gain.

Step 2.1 Measurement: Measure the wind speeds within the time period $T_{w}$ and calculate the corresponding mean wind speed $v_{m}$ and turbulence intensity $T_{i}(k)=\sigma(k) / v_{m}(k)$, where $\sigma(k)$ and $v_{m}(k)$ are the standard deviation and mean values of the wind speeds within control period $T_{w}$, respectively.

Step 2.2 Mapping: Map the optimal gain coefficient $K_{\text {dopt }}$ via $K_{\text {dopt }}=f\left(v_{m}, T_{i}\right)$ and execute the optimal control variable, e.g., the electrical toque $T_{e}$.

Step 2.3 Updating: After the control period $T_{k}$, return to Step 2.1 and let $k=k+1$.

The proposed IATG control considers changing wind speeds and addresses the issue of losing tractability of the ATG control. Specifically, the IATG makes use of off-line learning and on-line optimization, and improves operational efficiency.

\section{Simulation Results}

In this section, the proposed IATG control is tested through simulation, and its performances are compared with the conventional ATG control.

The simulation parameters of the WECS models are listed in Table $3[18,29,30]$. In addition, parameters of the control methods are consistent with each other. The gain coefficient of the DTG control is set as 0.8 as it in [18], and $T_{w}$ and $T_{k}$ of the ATG control are the same with the IATG control.

Table 3. Simulation parameters.

\begin{tabular}{cc}
\hline WECS (Wind Energy Conversion System) & Parameters \\
\hline Air density $\varrho /\left(\mathrm{kg} / \mathrm{m}^{3}\right)$ & 1.225 \\
Wind turbine capacity $P_{\text {rat }}(\mathrm{MW})$ & 1.0 \\
Wind turbine radius $R(\mathrm{~m})$ & 26.335 \\
Wind turbine inertia $J /\left(\mathrm{kg} \cdot \mathrm{m}^{2}\right)$ & $1.1204 \times 10^{6}$ \\
Optimal tip-speed ratio $\lambda_{\text {opt }}$ & 8.0 \\
Maximum power coefficient $C_{\text {pmax }}$ & 0.4109 \\
\hline \multicolumn{2}{c}{ Control Parameters } \\
\hline$T_{w}=1 \mathrm{~s}, T_{k}=20$ min, and $\gamma_{k d}=1.4735$
\end{tabular}

\subsection{Off-Line Learning}

Following Steps 1.1 and 1.2, we generate 288 wind speed profiles and obtain the dataset of the average wind speed $v_{m}$, the turbulence intensity $T_{i}$, and the optimal gain $K_{\text {dopt }}$. Figure 8a shows that $K_{\text {dopt }}$ under different $v_{m}$ and $T_{i}$ presents trackable features. Some selected results of Figure $8 \mathrm{a}$ are shown in Figure $8 b, c$. It is observed that (1) the optimal gain $K_{\text {dopt }}$ under a fixed mean wind speed decreases with the turbulence intensity $T_{i}$ increasing; and (2) the $T_{i}-K_{\text {dopt }}$ curves under different mean wind speeds have strong similarities. 


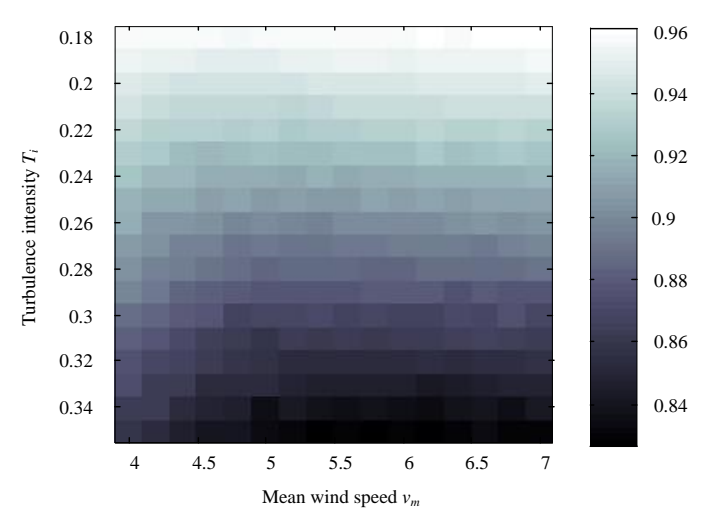

(a)
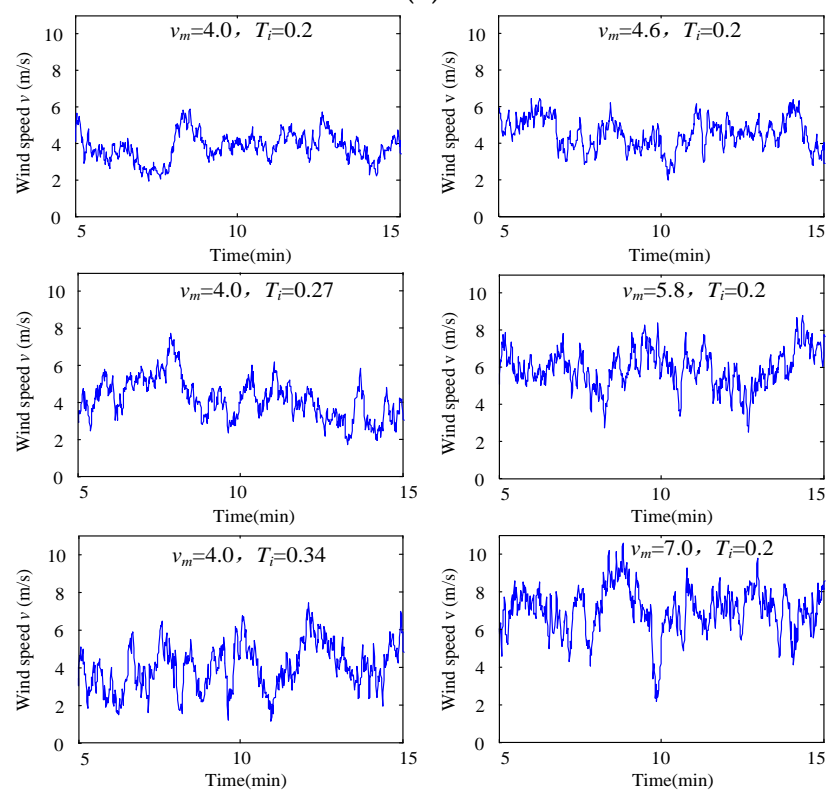

(b)

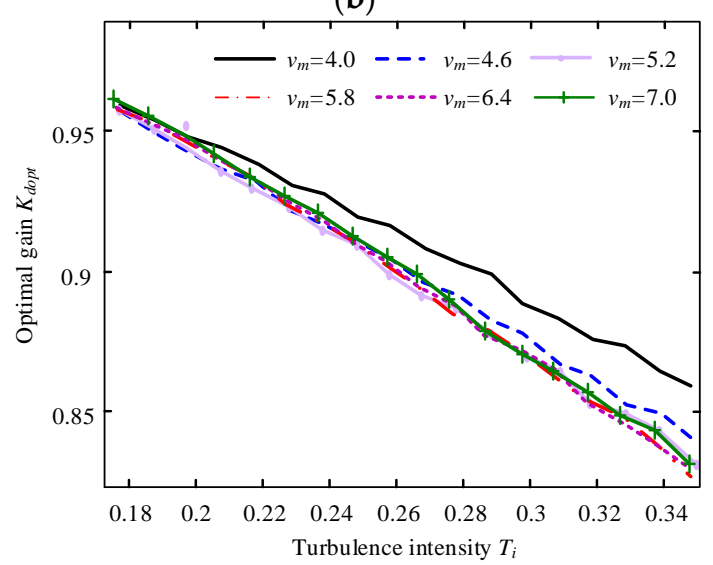

(c)

Figure 8. Results of off-line learning: (a) optimal gains with different mean wind speeds and turbulence intensities; (b) selected results of inputs (i.e., wind speed with different mean values and turbulence intensities); and (c) selected results of outputs (i.e., optimal gains with fixed mean wind speeds and variable turbulence intensities).

The relationship between $v_{m}, T_{i}$, and $K_{\text {dopt }}$ is further analyzed using standard deviation. Here, we fix each mean wind speed and calculate the standard deviation of $K_{\text {dopt }}$ under different $T_{i}$; then, we fix each turbulence intensity and calculate the standard deviation of $K_{\text {dopt }}$ under different $v_{m}$. 
The statistical results of the standard deviation are shown in Figure 9 and Table 4. It is found that the truculence intensity has a greater impact on the optimal gain, suggesting that the truculence intensity plays a decisive role in determining the optimal gains. Hence, the $T_{i}-v_{m}-K_{\text {dopt }}$ curve is simplified as the $T_{i}-K_{\text {dopt }}$ curve.

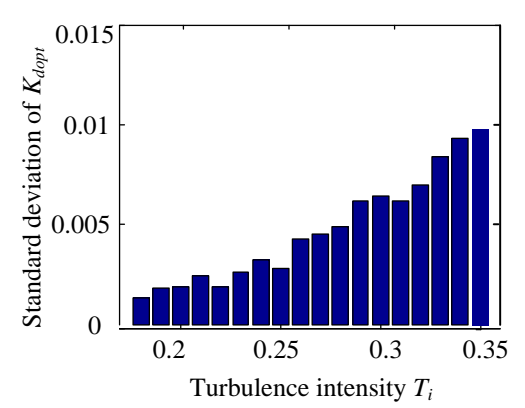

(a)

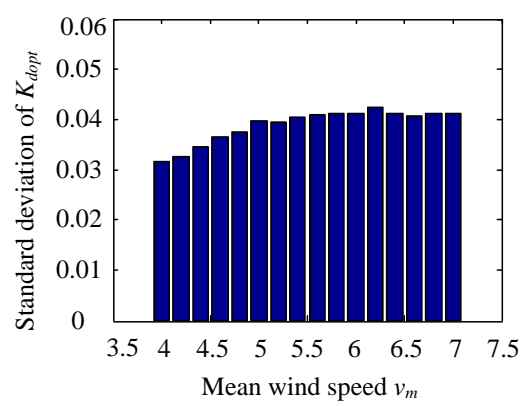

(b)

Figure 9. Statistical results of the relationship of mean wind speeds, turbulence intensities, and optimal gains: (a) standard deviation of optimal gains with fixed mean wind speeds and variable turbulence intensities; and (b) standard deviation of optimal gains with variable mean wind speeds and fixed turbulence intensities.

Table 4. Statistical results of the relationship of mean wind speeds, turbulence intensities, and optimal gains.

\begin{tabular}{ccc}
\hline Mean Wind Speed & Turbulence Intensity & Average $\boldsymbol{K}_{\text {dopt }}^{\text {std }}$ \\
\hline Fixed & Varying & 0.0389 \\
Varying & Fixed & 0.0047 \\
\hline
\end{tabular}

Next, following Step 1.3, a series of $T_{i}-K_{\text {dopt }}$ curves are drawn in Figure 10, and their functional relationship is approximated using the least squares algorithm. Note that the $T_{i}-K_{\text {dopt }}$ curve with the averaged $v_{m}$ is used in the least squares algorithm, which efficiently integrates the effect of mean wind speeds and turbulence intensities. Specifically, compared with the cubic-fitting and quantic-fitting based least square algorithms, the one-time fitting algorithm achieves favorable accuracy and demands less hardware cost. Therefore, the one-time-fitting based least square algorithm is used here, and the functional relationship of $K_{\text {dopt }} \& T_{i}$ is obtained in Equation (13) with the fitting error $2.10 \times 10^{-5}$ :

$$
K_{\text {dopt }}=-0.7228 \times T_{i}+1.0888
$$

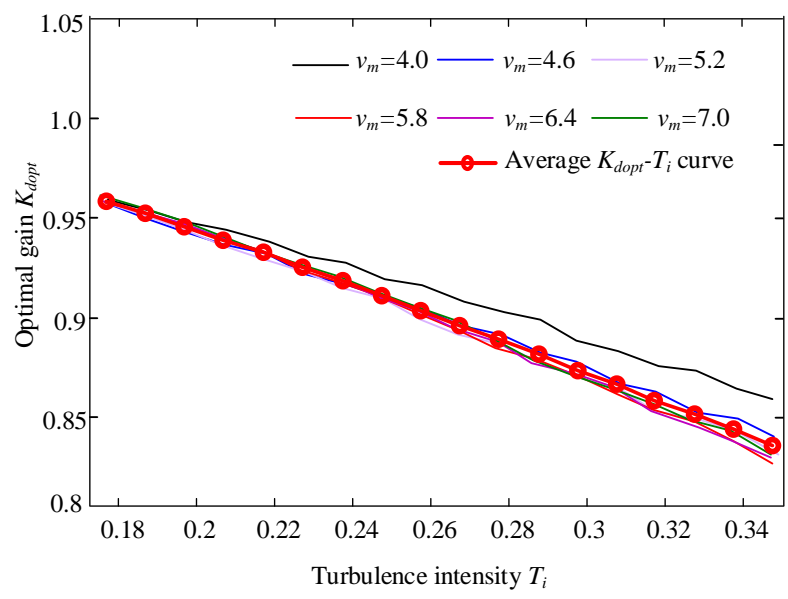

Figure 10. $T_{i}-K_{\text {dopt }}$ curves under different mean wind speeds. 


\subsection{On-Line Optimization}

With the knowledge obtained from the off-line analysis, the proposed IATG control is performed following Steps 2.1-2.3. The performance of the proposed control is tested as follows.

First, the performances of the ATG control and IATG control are compared. To be specific, 150 groups of wind speed profiles over $150 \times 600 \mathrm{~min}$ are selected from a wind farm, covering class A, class $B$, and class $C$ turbulence categories. In each wind speed profile, the ATG control and IATG control run 30 times over $600 \mathrm{~min}$, and the real optimal gain under each wind speed is calculated through traversal. Consequently, a group of searching results of the ATG control and IATG control are shown in Figures 11-13, and the 150 groups of searching results total are presented in a statistical way as shown in Figure 14 and Table 5. Note that $\bar{e}_{k d}$ denotes the average deviation of $e_{k d}$ and $P_{\text {wrong }}$ denotes the probability of incorrect searching directions. Figures 11-13 show that the IATG control searches the optimal gain more effectively than the ATG control. Figure 14 and Table 5 further display that the IATG control results in fewer searching errors, and the searching error is mainly distributed around zero. Thus, the IATG control presents better performance in searching the optimal control gains.

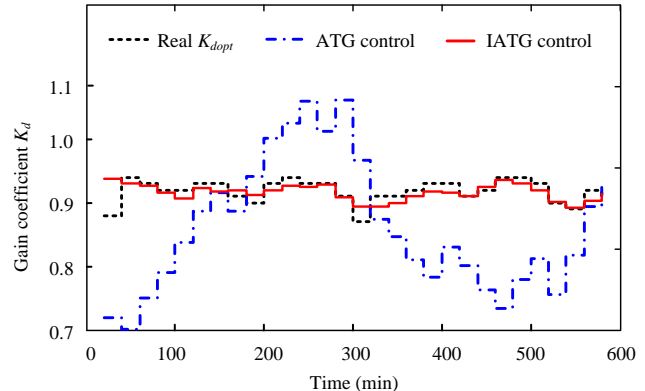

(a)

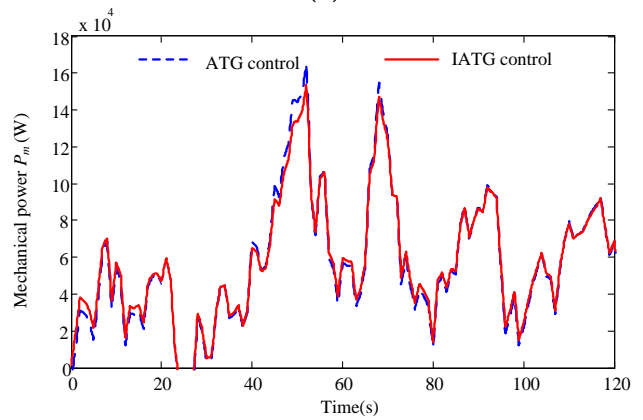

(c)

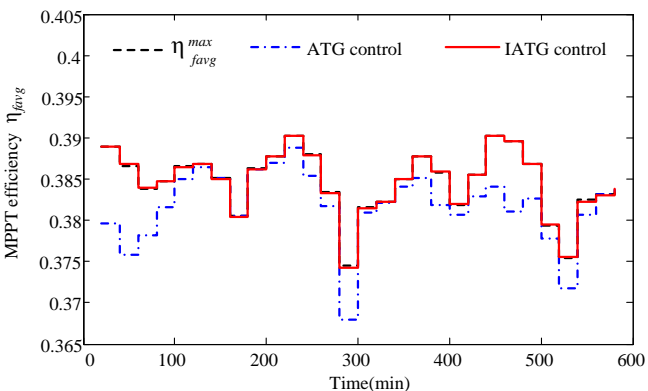

(b)

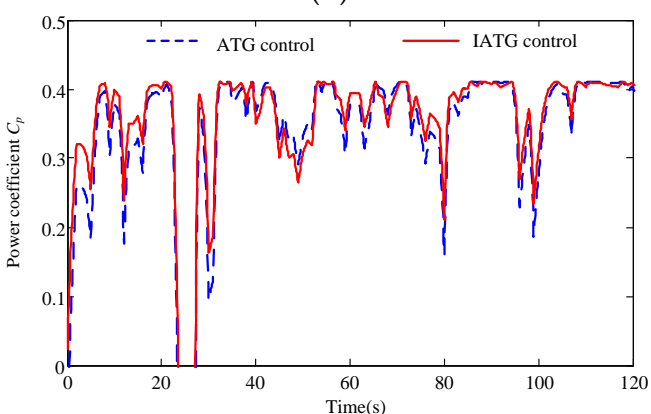

(d)

Figure 11. MPPT performances in searching the optimal gains under wind speed with $T_{i}$ of category A: (a) $K_{d}$ of ATG and IATG control; (b) MPPT efficiency $\eta_{f a v g}$; (c) mechanical power $P_{m}$; and (d) power coefficient $C_{p}$.

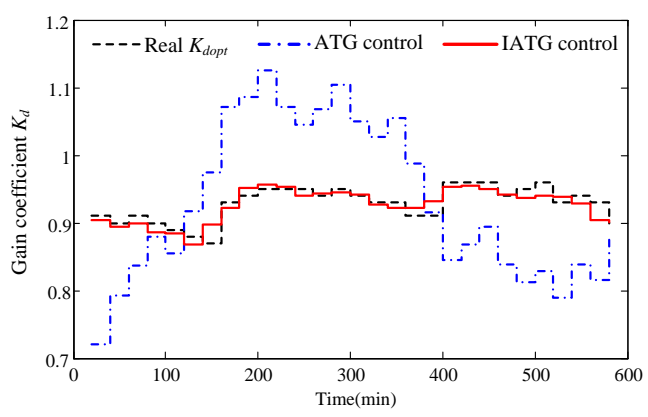

(a)

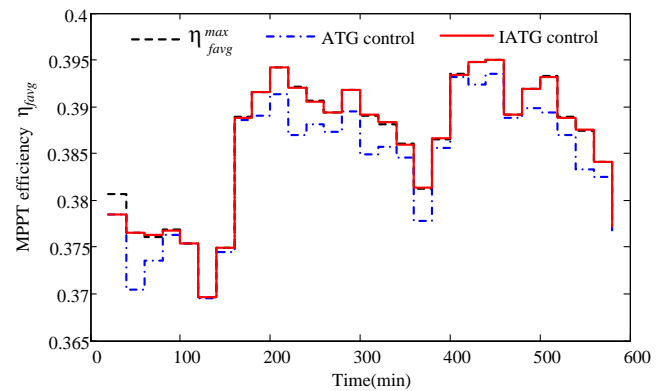

(b)

Figure 12. Cont. 


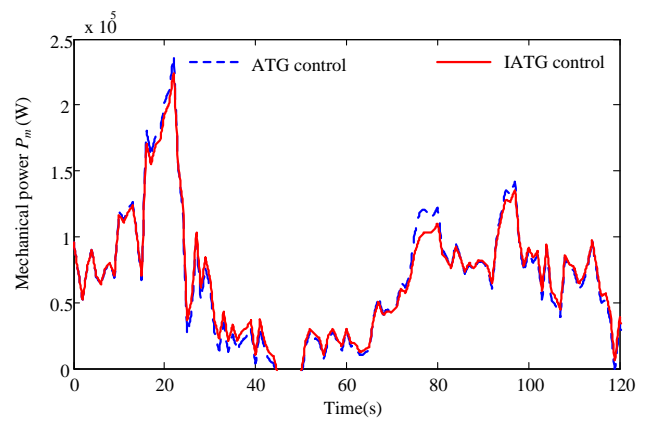

(c)

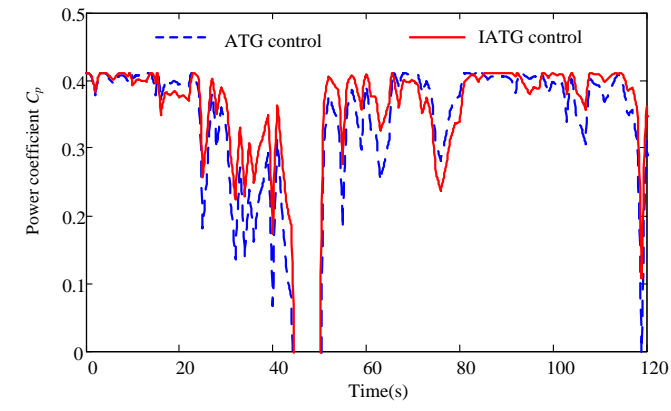

(d)

Figure 12. MPPT performances in searching the optimal gains under wind speed with $T_{i}$ of category B: (a) $K_{d}$ of ATG and IATG control; (b) MPPT efficiency $\eta_{f a v g} ;$ (c) mechanical power $P_{m}$; and (d) power coefficient $C_{p}$.

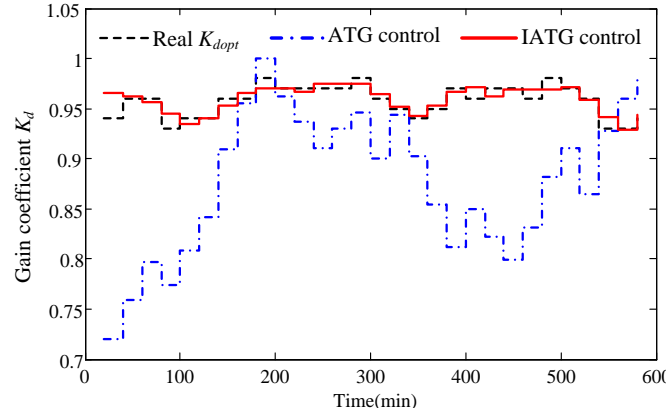

(a)

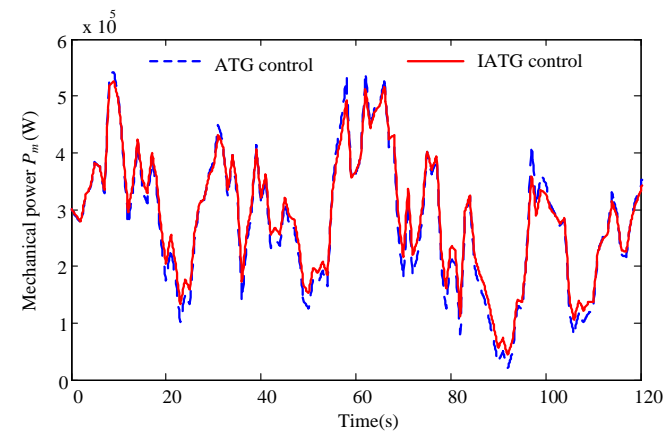

(c)

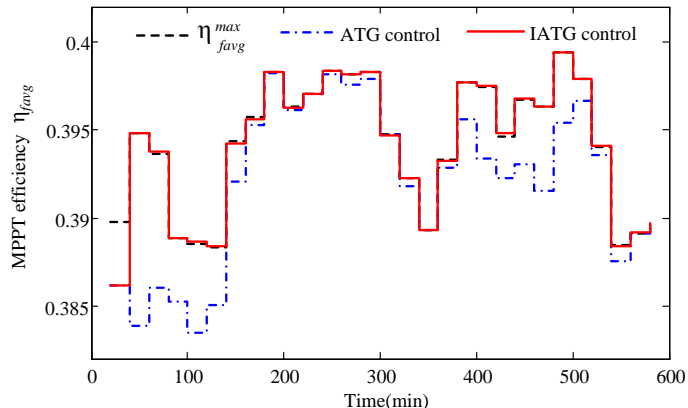

(b)

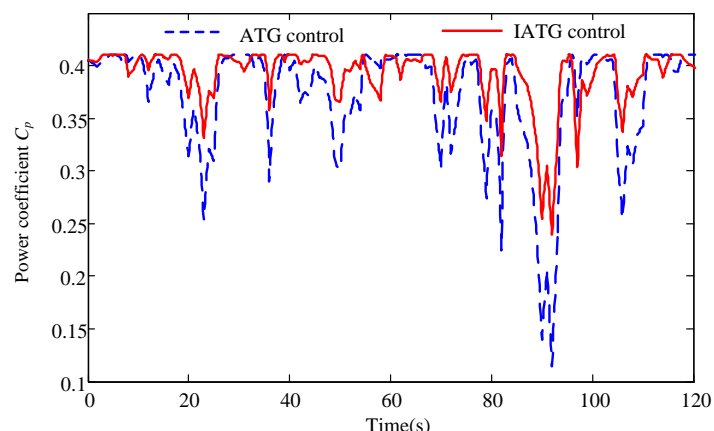

(d)

Figure 13. MPPT performances in searching the optimal gains under wind speed with $T_{i}$ of category C: (a) $K_{d}$ of ATG and IATG control; (b) MPPT efficiency $\eta_{\text {favg }}$; (c) mechanical power $P_{m}$; and (d) power coefficient $C_{p}$.

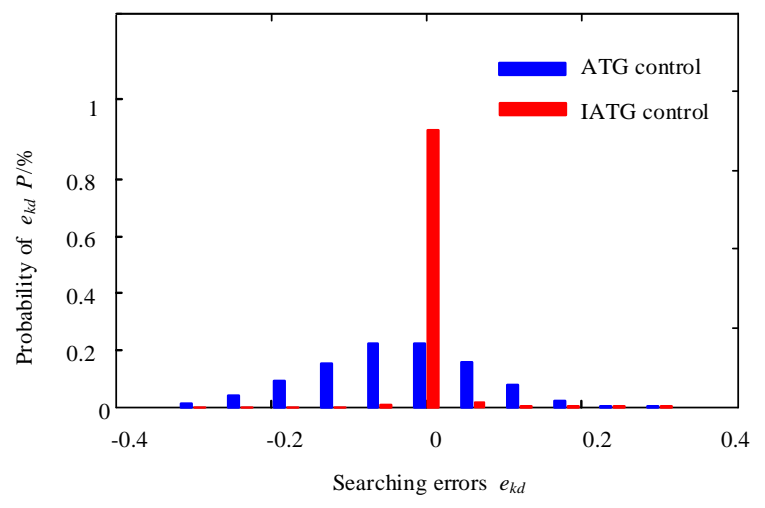

Figure 14. Distribution of the searching errors. 
Table 5. Statistical results of incorrect searching.

\begin{tabular}{ccc}
\hline MPPT Methods & $\overline{\boldsymbol{e}}_{\boldsymbol{k} \boldsymbol{d}}$ & $\boldsymbol{P}_{\text {wrong }}$ \\
\hline Adaptive torque gain control & 0.0861 & $44.55 \%$ \\
Improved adaptive torque gain control & 0.0091 & $32.30 \%$ \\
\hline
\end{tabular}

In addition, the performance of the IATG control is also tested in terms of the MPPT efficiency (i.e., fractional average power). The 150 groups of $600 \mathrm{~min}$ wind speed profiles are also used, and the PSF control, DTG control, ATG control, reducing tracking range (RTR) MPPT control [30], and the proposed IATG control are performed, respectively. Their performances are evaluated with the average results of fractional average power as follows:

$$
\bar{\eta}_{\text {favg }}=\sum_{i=1}^{n_{d}} \eta_{\text {favgi }} / n_{d}
$$

where $n_{d}$ is the total iteration number over the whole wind speed profiles.

Furthermore, the average value of $\bar{\eta}_{\text {favg }}$ from 150 simulation cases is denoted as $\eta_{\text {favg }}^{\text {avg }}$ and the maximum fractional average power of real $K_{d o p t}$ is denoted as $\eta_{f a v g}^{\max }$, respectively. Thus, the improvements of the proposed control method over the convention four control methods can be evaluated with $p_{\text {favg }}$ as

$$
p_{\text {favg }}(i)=\left[\eta_{\text {favg }}^{a v g(0)}-\eta_{\text {favg }}^{a v g(i)}\right] / \eta_{\text {favg }}^{a v g(0)}, i=1,2, \cdots 5
$$

where $\eta_{f a v g}^{a v g(0)}$ is the fractional average power of the proposed method and $\eta_{\text {favg }}^{a v g(i)}$ represents the fractional average power of the previous methods (including $\eta_{\text {favg }}^{\max }$ ).

The results in Table 6 show that the fractional average power of the proposed method is higher than the conventional methods. Moreover, these results suggest that the MPPT efficiency of the proposed method is closer to the maximum MPPT efficiency, and the proposed IATG control can reduce energy loss.

Table 6. Improvements of IATG control over conventional control methods.

\begin{tabular}{ccc}
\hline MPPT Algorithms & $\eta_{\text {favg }}^{a v g}$ & $p_{\text {favg }}$ \\
\hline PSF & 0.3863 & $0.24 \%$ \\
DTG & 0.3840 & $0.85 \%$ \\
ATG & 0.3851 & $0.57 \%$ \\
RTR & 0.3843 & $0.78 \%$ \\
IATG & 0.3873 & -- \\
$\eta_{\text {favg }}^{\operatorname{ax}}$ & 0.3874 & $-0.03 \%$ \\
\hline
\end{tabular}

\section{Conclusions}

MPPT plays a critical role in enhancing WECS efficiency. However, the majority of conventional MPPT methods view MPPT as a static process and ignore the wind speed changing during the control period, which leads to significant wind energy loss. To address this issue, the improved MPPT control is proposed in this paper. The contribution of this paper can be summarized as follows:

First, the conventional MPPT methods are briefly reviewed, and the potential issues in the PSF control, DTG control, and ATG control are investigated. It is found that the ATG control is blind to the atmospheric changes, which may lead to incorrect tracking direction and MPPT failures. It is also revealed that the mistaken tracking is impacted by wind speed fluctuations.

Second, the IATG control is proposed to improve the ATG control, and its performance is demonstrated via simulation. The IATG control considers the wind speed fluctuations and updates 
the optimal control gains adaptively. Specifically, it makes use of the off-line learning and on-line optimization and obtains the functional relationship between $K_{\text {dopt }}$ and $T_{i}$. The $K_{\text {dopt }}-T_{i}$ curves can be recorded in a WECS easily, and thus the IATG control can be implemented quickly and simply.

Furthermore, MPPT in essence is a tracking problem and its success largely depends on a reasonable selection of tracking references. This paper works out the relationship between wind speed characteristics and optimal gain coefficients (i.e., tracking references), which has not been reported in previous literature. The proposed IATG control with the favorable tracking references can improve MPPT performances and WECS efficiency.

Further works may include implementing the proposed IATG control in a real WECS and investigating the impact of the IATG on wind farms and renewable energy integration.

Acknowledgments: This work was supported by the National Natural Science Foundation of China (51607083, 51607084), the Natural Science Foundation of Jiangsu Province (BK20160778), the Foundation of Nanjing Institute of Technology (YKJ201413, YKJ201412), and the Open Research Fund of Jiangsu Collaborative Innovation Center for Smart Distribution Network (No. XTCX201612). The authors would like to thank Fangxing Li at the University of Tennessee for providing suggestions on improving the quality of this work.

Author Contributions: Xiaolian Zhang and Can Huang conceived the idea and led the whole work. Sipeng Hao, Fan Chen, and Jingjing Zhai contributed simulation data and analysis.

Conflicts of Interest: The authors declare no conflict of interest.

\section{References}

1. Cheng, M.; Zhu, Y. The state of the art of wind energy conversion systems and technologies: A review. Energy Convers. Manag. 2014, 88, 332-347. [CrossRef]

2. Wu, P.; Xia, B.; Zhao, X. The importance of use and end-of-life phases to the life cycle greenhouse gas (GHG) emissions of concrete-A review. Renew. Sustain. Energy Rev. 2014, 37, 360-369. [CrossRef]

3. Kumar, D.; Chatterjee, K. A review of conventional and advanced MPPT algorithms for wind energy systems. Renew. Sustain. Energy Rev. 2016, 55, 957-970. [CrossRef]

4. Zhu, Y.; Cheng, M.; Hua, W.; Wang, W. A novel maximum power point tracking control for permanent magnet direct drive wind energy conversion systems. Energies 2012, 5, 1398-1412. [CrossRef]

5. Yang, Z.Q.; Yin, M.H.; Xu, Y.; Zhang, Z.Y.; Zou, Y.; Dong, Z.Y. A multi-point method considering the maximum power point tracking dynamic process for aerodynamic optimization of variable-speed wind turbine blades. Energies 2016, 9. [CrossRef]

6. Luo, X.; Niu, S.X. Maximum power point tracking sensorless control of an axial-flux permanent magnet vernier wind power generator. Energies 2016, 9. [CrossRef]

7. Ou, T.C.; Su, W.F.; Liu, X.Z.; Huang, S.J.; Tai, T.Y. A modified bird-mating optimization with hill-climbing for connection decisions of transformers. Energies 2016, 9. [CrossRef]

8. Lin, W.M.; Hong, C.M.; Ou, T.C.; Chiu, T.M. Hybrid intelligent control of PMSG wind generation system using pitch angle control with RBFN. Energy Convers. Manag. 2011, 52, 1244-1251. [CrossRef]

9. Hong, C.M.; Ou, T.C.; Lu, K.H. Development of intelligent MPPT (maximum power point tracking) control for a grid-connected hybrid power generation system. Energy 2013, 50, 270-279. [CrossRef]

10. Lin, W.M.; Ou, T.C. Unbalanced distribution network fault analysis with hybrid compensation. IET Gener. Transm. Distrib. 2011, 5, 92-100. [CrossRef]

11. Ou, T.C.; Hong, C.M. Dynamic operation and control of microgrid hybrid power systems. Energy 2014, 66, 314-323. [CrossRef]

12. Ou, T.C. A novel unsymmetrical faults analysis for microgrid distribution systems. Int. J. Electr. Power Energy Syst. 2012, 43, 1017-1024. [CrossRef]

13. Ou, T.C. Ground fault current analysis with a direct building algorithm for microgrid distribution. Int. J. Electr. Power Energy Syst. 2013, 53, 867-875. [CrossRef]

14. Ou, T.C.; Chuang, S.J.; Hong, C.M.; Wu, R.C.; Tsao, T.P.; Chen, C.Y. Self-regulation ground faults model for microgrid distribution. ICIC Express Lett. B Appl. 2015, 6, 1-6.

15. The Global Wind Energy Council: Global Wind Statistics 2015. Available online: http://www.gwec.net/wpcontent/uploads/vip/GWEC-PRstats-2015_LR.pdf (accessed on 9 October 2016). 
16. Guo, J.; You, S.; Huang, C.; Liu, H.; Chai, J.; Wu, L.; Zhou, D.; Liu, Y.; Glass, J. An ensemble photovoltaic power forecasting model through statistical learning of historical weather dataset. In Proceedings of the 2016 PES General Meeting, Boston, MA, USA, 17-21 July 2016; pp. 1-5.

17. Huang, C.; Li, F.; Ding, T.; Jin, Z.; Ma, X. Second-order cone programming-based optimal control strategy for wind energy conversion systems over complete operating regions. IEEE Trans. Sustain. Energy 2015, 6, 263-271. [CrossRef]

18. Johnson, K.E. Adaptive Torque Control of Variable Speed Wind Turbines; NREL/TP-500-36265; National Renewable Energy Laboratory: Golden, CO, USA, 2004.

19. Johnson, K.E.; Pao, L.Y.; Balas, M.J.; Fingersh, L.J. Control of variable-speed wind turbines: Standard and adaptive techniques for maximizing energy capture. IEEE Trans. Control Syst. 2006, 26, 70-81. [CrossRef]

20. Zhou, D.; Blaabjerg, F.; Franke, T.; Tonnes, M. Comparison of wind power converter reliability with low-speed and medium-speed permanent-magnet synchronous generators. IEEE Trans. Ind. Electron. 2015, 62, 6575-6584. [CrossRef]

21. Zhao, Y.; Wei, C.; Zhang, Z.; Qiao, W. A review on position/speed sensorless control for permanent-magnet synchronous machine-based wind energy conversion systems. IEEE J. Emerg. Sel. Top. Power Electron. 2013, 1, 203-216. [CrossRef]

22. Huang, C.; Li, F.; Jin, Z. Maximum power point tracking strategy for large-scale wind generation systems considering wind turbine dynamics. IEEE Trans. Ind. Electron. 2015, 62, 2530-2539. [CrossRef]

23. Kim, K.H.; Van, T.L.; Lee, D.C.; Song, S.H.; Kim, E.H. Maximum output power tracking control in variable-speed wind turbine systems considering rotor inertial power. IEEE Trans. Ind. Electron. 2013, 60, 3207-3217. [CrossRef]

24. Tang, C.; Soong, W.L.; Freere, P.; Pathmanathan, M.; Ertugrul, N. Dynamic wind turbine output power reduction under varying wind speed conditions due to inertia. Wind Energy 2013, 16, 561-573. [CrossRef]

25. Raza, K.S.M.; Goto, H.; Guo, H.J.; Ichinokura, O. A novel algorithm for fast and efficient speed-sensorless maximum power point tracking in wind energy conversion systems. IEEE Trans. Ind. Electron. 2011, 58, 29-36.

26. Johnson, K.E.; Fingersh, L.J.; Balas, M.J.; Pao, L.Y. Methods for increasing region 2 power capture on a variable-speed wind turbine. J. Sol. Energy Eng. 2004, 126, 1092-1100. [CrossRef]

27. Xia, Y.; Ahmed, K.H.; Williams, B.W. Wind turbine power coefficient analysis of a new maximum power point tracking technique. IEEE Trans. Ind. Electron. 2013, 60, 1122-1132. [CrossRef]

28. Xia, Y.; Ahmed, K.H.; Williams, B.W. A new maximum power point tracking technique for permanent magnet synchronous generator based wind energy conversion system. IEEE Trans. Power Electron. 2011, 26, 3609-3620. [CrossRef]

29. Yin, M.; Zhang, X.; Zou, Y.; Zhou, L. Improved MPPT control of wind turbines based on optimization of tracking range. Power Syst. Technol. 2014, 38, 2180-2185.

30. Yin, M.; Zhang, X.; Ye, X.; Zou, Y. Improved MPPT control based on the reduction of tracking range. Proc. CSEE 2012, 32, 24-31.

31. Ma, Z.; Shaltout, M.; Chen, D. An adaptive wind turbine controller considering both the system performance and fatigue loading. J. Dyn. Syst. Meas. Control 2015, 137. [CrossRef]

32. She, Y.; She, X.; Baran, M.E. Universal tracking control of wind conversion system for purpose of maximum power acquisition under hierarchical control structure. IEEE Trans. Energy Convers. 2011, 26, 766-775. [CrossRef]

33. Jin, Z.; Huang, C. DFIG voltage control based on dynamically adjusted control gains. J. Power Energy Eng. 2014, 2, 45-58. [CrossRef]

34. Blaabjerg, F.; Liserre, M.; Ma, K. Power electronics converters for wind turbine systems. IEEE Trans. Ind. Appl. 2011, 48, 281-290.

35. Chen, Z.; Guerrero, J.M.; Blaabjerg, F. A review of the state of the art of power electronics for wind turbines. IEEE Trans. Power Electron. 2009, 24, 1859-1875. [CrossRef]

36. Chen, J.; Chen, J.; Gong, C. Constant-bandwidth maximum power point tracking strategy for variable-speed wind turbines and its design details. IEEE Trans. Ind. Electron. 2013, 60, 5050-5058. [CrossRef]

37. Shokrzadeh, S.; Jozani, M.J.; Bibeau, E. Wind turbine power curve modeling using advanced parametric and nonparametric methods. IEEE Trans. Sustain. Energy 2014, 5, 1262-1269. [CrossRef] 
38. Jin, Z.; Li, F.; Ma, X.; Djouadi, S.M. Semi-definite programming for power output control in wind energy conversion system. IEEE Trans. Sustain. Energy 2014, 5, 466-475. [CrossRef]

39. Fan, L.; Miao, Z. Modeling and Analysis of Doubly Fed Induction Generator Wind Energy Systems; Elsevier Science \& Technology Books; Elsevier: Amsterdam, The Netherlands, 2015.

40. Bianchi, F.D.; Battista, H.; Mantz, R.J. Wind Turbine Control Systems: Principles, Modeling and Gain Scheduling Design; Springer: New York, NY, USA, 2007.

41. Nichita, C.; Luca, D.; Dakyo, B.; Ceanga, E. Large band simulation of the wind speed for real time wind turbine simulators. IEEE Trans. Energy Convers. 2002, 17, 523-529. [CrossRef]

(C) 2016 by the authors; licensee MDPI, Basel, Switzerland. This article is an open access article distributed under the terms and conditions of the Creative Commons Attribution (CC-BY) license (http://creativecommons.org/licenses/by/4.0/). 